\title{
The effect of gestational diabetes mellitus on sufentanil consumption after cesarean section: a prospective cohort study
}

\author{
Chen Yang, Wei Lian Geng, Jianying Hu and Shaoqiang Huang * (D)
}

\begin{abstract}
Background: Previous studies have shown that patients with long-term diabetes require more opioids after surgery than patients without diabetes. Gestational diabetes mellitus (GDM) normally only lasts for a brief period; nevertheless, its effect on sufentanil consumption after cesarean section is unknown.

Methods: This prospective cohort study included two groups: a GDM group $(n=32)$ and a matched non-GDM (NGDM) group $(n=32)$. All patients underwent routine combined spinal-epidural anesthesia for cesarean delivery. Sufentanil consumption through an intravenous patient-controlled analgesia (PCA) pump, the frequency of PCA requests, and visual analog scale (VAS) scores 6 and $24 \mathrm{~h}$ after surgery were compared between groups.
\end{abstract}

Results: Sufentanil consumption $(\mu \mathrm{g}) 6 \mathrm{~h}$ after surgery was higher in the GDM group than in the NGDM group (24.0 \pm 6.6 vs $20.1 \pm 5.7, P=0.023)$. PCA was used more frequently 6 and $24 \mathrm{~h}$ after surgery by the GDM group than by the NGDM group (1[0-2] vs $0[0-1], P=0.001 ; 6[1-5]$ vs $3[1,2,6-8], P=0.001$, respectively). The VAS score during activity $24 \mathrm{~h}$ after surgery was higher in the GDM group than in the NGDM group (5 [2, 3] vs $5[1,2]$, respectively, $P=0.03$ ).

Conclusion: Pregnant women with GDM require more opioids during the immediate postoperative period after cesarean section than those without GDM.

Clinical trials registration: No. ChiCTR1800016014, ChenYang, May 6th 2018.

Keywords: Gestational diabetes mellitus, Postoperative analgesia, Cesarean section

\section{Background}

Gestational diabetes mellitus (GDM) is defined as high blood glucose caused by impaired glucose tolerance detected and diagnosed during pregnancy. GDM is characterized by elevated fasting blood glucose and impaired glucose tolerance during pregnancy. The reference values used in the oral glucose tolerance test for pregnant women are as follows: fasting, $5.6 \mathrm{mmol} / \mathrm{L} ; 1$-h postprandial, $10.3 \mathrm{mmol} / \mathrm{L} ; 2$-h postprandial, $8.6 \mathrm{mmol} / \mathrm{L} ; 3$ - $\mathrm{h}$ postprandial, $6.7 \mathrm{mmol} / \mathrm{L}$. GDM is diagnosed when two or more test values reach or exceed the reference values. In most cases, GDM resolves 1 to 2 months after delivery ("transient diabetes"). GDM is one of the most common complications of pregnancy, with a prevalence of approximately 3 to $7 \%$ [6-8].

\footnotetext{
* Correspondence: drhuangsq@163.com

Department of Anesthesiology, Obstetrics \& Gynecology Hospital, Fudan University, 128\# Shenyang road, Shanghai 200090, China
}

Karci et al. showed that the analgesic effect of morphine is negatively affected by high blood glucose in patients scheduled for elective total abdominal hysterectomy. According to data on postoperative intravenous patientcontrolled analgesia (PCA), patients with diabetes require more opioids than patients without diabetes [1]. Glycated hemoglobin (HbA1c) is a highly reliable indicator of glycemic control over the previous 8 to 12 weeks [2]. Kim et al. conducted a prospective observational study and found that perioperative $\mathrm{HbA} 1 \mathrm{c}$ was positively correlated with postoperative opioid (fentanyl) consumption among patients with diabetes undergoing open nephrectomy [3]. A retrospective cohort study conducted by Weiner et al. showed that total opioid consumption during the 4month period following surgery was increased in diabetics with operative ankle fractures [4]. However, the mechanism that underlies this relationship is unknown. Chronic high blood glucose might affect opioid receptors, thereby

(c) The Author(s). 2020 Open Access This article is distributed under the terms of the Creative Commons Attribution 4.0 International License (http://creativecommons.org/licenses/by/4.0/), which permits unrestricted use, distribution, and 
altering the pharmacokinetics and pharmacodynamics of opioids [5]. Alternatively, chronic high blood glucose might affect a patient's metabolism [9] or neurotransmitter levels [10-12].

The patients included in previous studies had been diagnosed with diabetes for at least 60 weeks [3]; however, the course of gestational diabetes is usually shorter than this, and its association with opioid consumption is unclear. Therefore, we conducted a prospective observational cohort study in which we investigated the correlation between gestational diabetes and sufentanil consumption during the immediate postoperative period after cesarean section. We hypothesized that women with GDM require more opioids during the immediate postoperative period after cesarean section than those without GDM.

\section{Methods}

\section{Participants}

The Ethics Committee of the Obstetrics and Gynecology Hospital, Fudan University, approved this prospective, observational cohort study, and it was registered at the Clinical Trials Registry (http://www.chictr.org.cn/, Registration No. ChiCTR1800016014). This study was conducted between June 2018 and October 2018 at the Obstetrics and Gynecology Hospital, Fudan University. Inclusion criterion: pregnant women, American Society of Anesthesiology (ASA) II with a single fetus scheduled to undergo elective cesarean section under combined spinalepidural anesthesia. Exclusion criteria: history of opioid allergies, history of opioid use within the previous week, contraindications for spinal anesthesia, known DM or other pregnancy complications (e.g., gestational hypertension, pregnancy complicated with hypothyroidism, and preeclampsia). Pregnant women with GDM who met the above criteria were included in the GDM group. For each pregnant woman included in the GDM group, a pregnant woman without GDM who matched with respect to height $\pm 2 \mathrm{~cm}$, weight $\pm 1 \mathrm{~kg}$, and the same parity was included in the nonGDM (NGDM) group (Fig. 1). Patients signed an informed consent document prior to participation in the study.

\section{Procedure}

On the morning of cesarean section, blood was drawn for measurement of maternal glucose and HbAlc. Maternal age, height, weight, gestational age, and parity data were recorded. In addition, the blood glucose management methods used by patients in the GDM group, such as diet restrictions, oral medications (and doses), and insulin injections (and doses), were recorded.

No medication was given before the operation. In the operating room, an 18-gauge needle was used to puncture a vein in the right upper arm, and an indwelling catheter was placed. Blood pressure, electrocardiograms
(ECGs), heart rate, and pulse oximetry were routinely monitored noninvasively. Baseline values were recorded. At the beginning of anesthesia, 6\% hydroxyethyl starch was infused at $20 \mathrm{~mL} / \mathrm{min}$ until delivery. The infusion speed was then adjusted by the anesthesiologist according to the maternal circulation state until the total amount of $500 \mathrm{ml}$ hydroxyethyl starch had been infused and replaced with Ringer's lactate solution. A combined spinal-epidural anesthesia was performed at the L3-4 or L2-3 vertebral interspace with the patient in the left lateral position. An 18-gauge Tuohy needle was placed in the epidural space and advanced until there was loss of resistance to saline; then, a 25 -gauge Whitacre spinal needle was inserted through the Tuohy needle until the dura mater was punctured. Next, $8 \sim 10 \mathrm{mg}$ bupivacaine was diluted to $3 \mathrm{ml}$ with cerebrospinal fluid for intrathecal injection, and an epidural catheter $(3-4 \mathrm{~cm})$ was immediately placed. The patient was placed in the supine position, and the operating table was tilted to the left. During the first $10 \mathrm{~min}$ after spinal anesthesia administration, a needle was used to test the sensory block level every $2 \mathrm{~min}$. The operation began when the block level reached T6. Patients who did not achieve this level were excluded from the study, and $1.5 \%$ lidocaine was injected epidurally until successful anesthesia was achieved. During the operation, the patient received continuous supplementary oxygen through a mask at $5 \mathrm{~L} /$ min. If hypotension occurred (i.e., systolic blood pressure $<90 \mathrm{mmHg}$ or a decrease of $>20 \%$ from baseline), $40 \mu \mathrm{g}$ of phenylephrine was given intravenously (iv) and repeated as needed; furthermore, the infusion rate of hydroxyethyl starch was increased. If sinus bradycardia occurred (heart rate $<50 \mathrm{bpm}$ ), $0.2 \mathrm{mg}$ of atropine was given iv, and this was repeated as needed. If pain occurred after delivery but during the operation, it was treated with intravenous analgesics.

After the infant was delivered and the umbilical cord had been clamped, $50 \mathrm{mg}$ of flurbiprofen and $4 \mathrm{mg}$ of ondansetron were administered intravenously. At the end of the operation, $5 \mu \mathrm{g}$ of sufentanil (diluted in saline to $5 \mathrm{~mL}$ ) was given via epidural injection, and the epidural catheter was removed. The operative time and blood loss were recorded.

After the operation, the patient was moved to the post anesthesia care unit (PACU). When the patient's blood pressure and heart rate were normal and the anesthesia level was T6 or below, an intravenous analgesia pump (Aipeng, Nantong Apon Medical Devices Co., Ltd.) was connected, and the patient was instructed in its proper use. The analgesics given via patient-controlled intravenous analgesia (PCIA) included sufentanil $150 \mu \mathrm{g}$ and ondansetron $4 \mathrm{mg}$ diluted in saline to $150 \mathrm{~mL}$; the sufentanil concentration was $1 \mathrm{mg} / \mathrm{ml}$. The background dose was $3 \mathrm{~mL} / \mathrm{h}$, with a bolus dose of $3 \mathrm{~mL}$ and a lock-out 


\section{Pregnant women with a single fetus scheduled to undergo cesarean section under spinal-epidural anesthesia $(n=260)$}

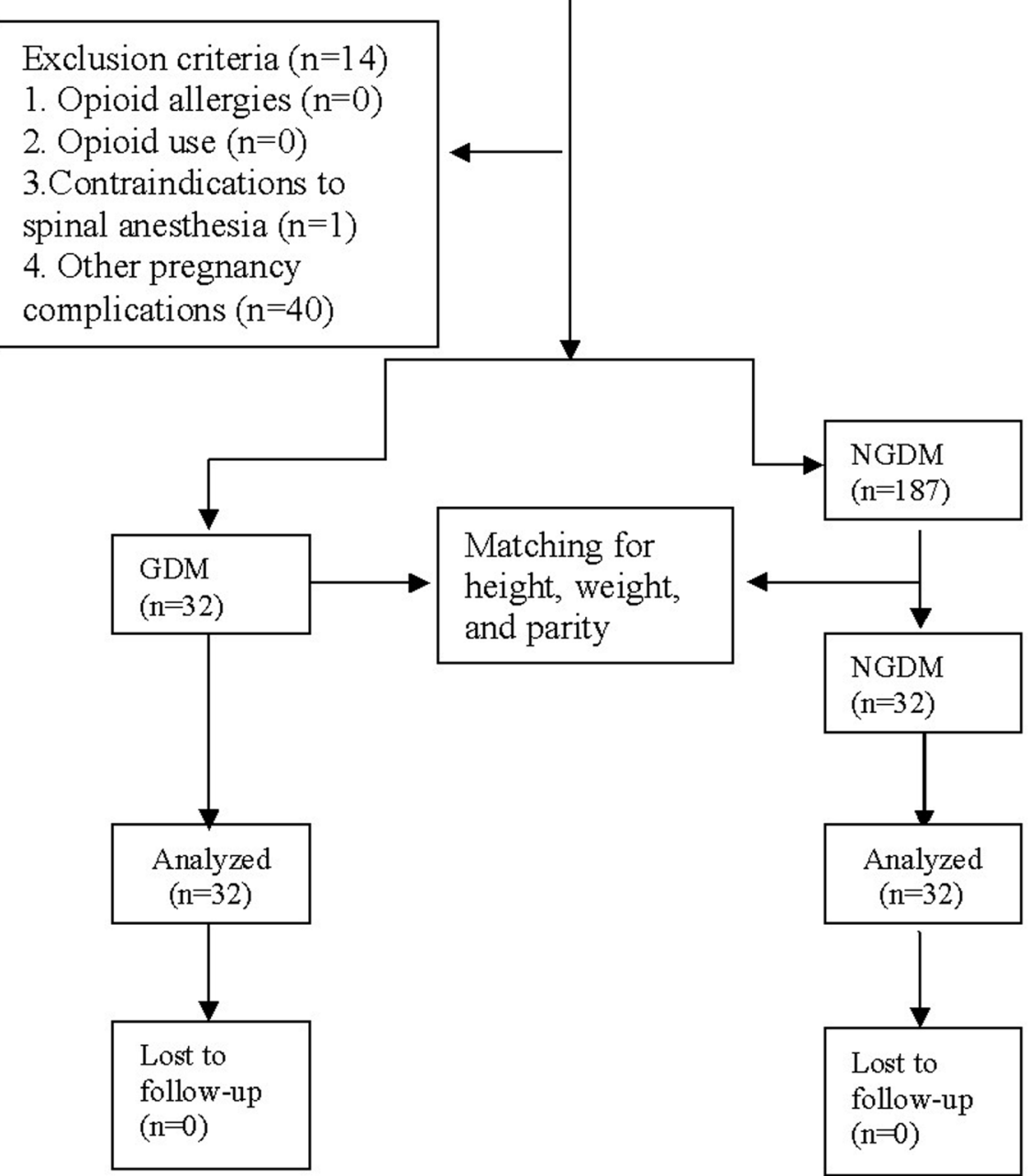

Fig. 1 Flow chart for patient enrolment

time of $15 \mathrm{~min}$. No other postoperative analgesics were administered during the study period. The anesthesia nurse involved in the study recorded the use of the postoperative analgesia pump (sufentanil consumption and number of PCA compressions [reflecting maternal need]) as well as adverse reactions such as nausea, vomiting, and itching. Patients with nausea and vomiting were given ondansetron $(4 \mathrm{mg}$, iv), which was repeated as needed. Moreover, a visual analog scale (VAS, $0 \mathrm{~cm} \sim$ $10 \mathrm{~cm}$ ) was used to assess pain during rest and activity 6 and $24 \mathrm{~h}$ after the operation. In addition, patient satisfaction with the postoperative analgesia was evaluated as 1 (very dissatisfied), 2 (dissatisfied), 3 (neither dissatisfied nor satisfied), 4 (satisfied), or 5 (very satisfied). Patients were excluded from this study if general anesthesia or intraoperative intravenous opioids were used, if other medications were administered intraoperatively through the epidural, if they underwent hysterectomy due to bleeding or for other reasons, if they discontinued the analgesia pump for any reason (e.g., severe surgical or medical complications), or if they asked to be withdrawn from the study early.

\section{Statistical analysis}

The primary endpoint was sufentanil consumption $6 \mathrm{~h}$ after the operation. The secondary endpoints included sufentanil consumption $24 \mathrm{~h}$ after the operation, the frequency of PCA press 6 and $24 \mathrm{~h}$ after the operation, the VAS score, adverse reactions during postoperative analgesia administration, and patient satisfaction with postoperative analgesia. The Kolmogorov-Smirnov test was performed to determine whether the data displayed a 
normal distribution. Normally distributed measurement data are expressed as the mean \pm standard deviation and analyzed using an independent-samples t-test. Categorical data (e.g., nausea, vomiting, and itching) were analyzed using Fisher's exact test. SPSS v22.0 (SPSS Inc., Chicago, IL, USA) was used for all analyses, and $P<0.05$ was considered significant. A multivariate analysis of covariance was performed to eliminate the offset effect of the statistically significant variables in the two groups on the results.

The primary endpoint was sufentanil consumption $6 \mathrm{~h}$ after the operation. We referenced a previous study that used the same analgesia regimen used in this study to estimate the necessary sample size [13]; in that study, NGDM patients used $15.8 \pm 6.3 \mu \mathrm{g}$ of sufentanil $6 \mathrm{~h}$ after the operation. We determined that the difference between GDM patients and NGDM patients must be at least $20 \%(4.6 \mu \mathrm{g})$ to reach clinical significance. With two-tailed tests, $\alpha=$ 0.05 and $\beta=0.2$, and at least 29 patients were required in each group. Ultimately, 32 patients were included in each group, allowing for a $10 \%$ attrition rate.

\section{Results}

All patients (32 in each group) completed this study (Fig. 1). The demographic and intraoperative characteristics of the patients are shown in Table 1 . The women in the GDM group were older and had a lower mean gestational time than those in the NGDM group.

Preoperative laboratory tests showed that fasting blood glucose $(\mathrm{mmol} / \mathrm{l})$ was higher in the GDM group than in the NGDM group, despite blood glucose management in the GDM group (diet restriction, medication, and insulin). HbA1c was significantly higher in the GDM group than in the NGDM group. No significant between-group differences were observed with regard to other indicators.

The data on analgesic use are shown in Table 2. The GDM group used the analgesia pump more frequently than did the NGDM group 6 and $24 \mathrm{~h}$ after the operation

Table 1 Clinical characteristics of the patients

\begin{tabular}{llll}
\hline & GDM $(n=32)$ & NGDM $(n=32)$ & $P$ \\
\hline Age (years) & $34.3 \pm 0.1$ & $32.1 \pm 0.1$ & 0.04 \\
Gestation (weeks) & $37.9 \pm 1.2$ & $38.8 \pm 1.2$ & 0.004 \\
Height (cm) & $162.1 \pm 3.2$ & $160.9 \pm 4.0$ & 0.24 \\
Weight (kg) & $69.3 \pm 8.5$ & $69.8 \pm 8.6$ & 0.48 \\
BMI (kg/m $\left.{ }^{2}\right)$ & $26.1 \pm 3.3$ & $26.9 \pm 3.1$ & 0.24 \\
Parity (first/repeat) & $14 / 18$ & $14 / 18$ & 1 \\
Amount of bleeding (ml) & $310 \pm 74$ & $315 \pm 70$ & 0.34 \\
Duration of surgery (min) & $45.3 \pm 4.2$ & $46.5 \pm 4.7$ & 0.32 \\
Blood glucose (mmol/l) & $4.8 \pm 0.2$ & $4.3 \pm 0.4$ & 0.0001 \\
HbA1c (\%) & $5.9 \pm 1.9$ & $4.9 \pm 0.3$ & 0.003 \\
Newborn weight(g) & $3146 \pm 214.8$ & $3296 \pm 149.2$ & 0.21 \\
\hline
\end{tabular}

Data are presented as the mean \pm SD or number
$(P<0.005)$. Sufentanil consumption $(\mu \mathrm{g}) 6 \mathrm{~h}$ after the operation was higher in the GDM group than in the NGDM group ( $24.0 \pm 6.6$ vs. $20.1 \pm 5.7, P=0.023)$. Sufentanil consumption $24 \mathrm{~h}$ after the operation in the GDM group and the NGDM group did not differ significantly.

The results of multivariate analysis of covariance showed that age $(F=0.893, P=0.348)$ and gestational week $(F=0.005, P=0.944)$ had no significant effect on opioid consumption $6 \mathrm{~h}$ after surgery. After controlling for age and gestational week, opioid consumption $6 \mathrm{~h}$ after surgery was still significantly higher in the GDM group of pregnant women $(24.2 \mu \mathrm{g}, 95 \% \mathrm{CI}: 21.7-$ $26.6 \mu \mathrm{g})$ than in the NGDM group $(20.1 \mu \mathrm{g}, 95 \% \mathrm{CI}$ : $17.6-22.5 \mu \mathrm{g})(F=5.097, P=0.028)$. The difference in opioid consumption between the groups was $4.1 \mu \mathrm{g}(95 \%$ CI: 0.5-7.7 $\mu \mathrm{g}$ ) (Table 3).

The VAS scores during rest and activity were assessed 6 and $24 \mathrm{~h}$ after the operation (Table 2). The VAS score during activity $24 \mathrm{~h}$ after the operation was higher for the GDM group than for the NGDM group $(5[2,3]$ vs $5[1,2], P=$ $0.03)$. No significant between-group differences were found in the pain scores at the 6-h postoperative rest/activity measurement or at the 24-h postoperative rest measurement.

No significant between-group differences were observed in adverse reactions during postoperative analgesia administration or with regard to patient satisfaction with postoperative analgesia (Table 4).

\section{Discussion}

This prospective cohort study showed that pregnant women with GDM require more opioids and exhibit higher sufentanil consumption $6 \mathrm{~h}$ after cesarean section than NGDM patients.

In animal models of diabetes mellitus (DM), high blood glucose has been shown to reduce the effectiveness of opioid receptor agonists [14-16]. Studies on relevant mechanisms have shown that high blood glucose is associated with changes in the expression of opioid receptor genes [17, 18], the body's metabolism [9], and neurotransmitter levels $[11,12]$.

Previous studies were conducted in patients who had displayed elevated blood glucose (diabetes) for more than 60 weeks $[1,3]$. In contrast, this study was conducted among pregnant women who were diagnosed with GDM after an abnormal glucose tolerance test at approximately 24 gestational weeks, and women with pre-GDM were excluded. As a result, the course of high blood glucose was only approximately 100 days, far briefer than that in a typical patient with diabetes. Nevertheless, this study showed that GDM patients consumed significantly more opioids $6 \mathrm{~h}$ after surgery than NGDM patients. The results are similar to those of previous studies of DM patients $[1,3]$. This finding is important because few previous studies have focused on 
Table 2 Postoperative analgesia

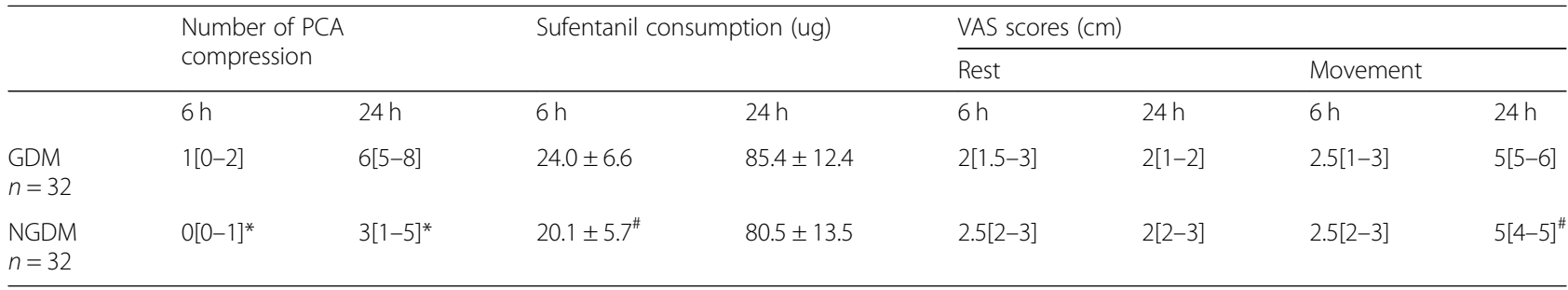

Data are presented as the mean \pm SD or median [IQR]. *P $<0.005$ vs GDM; ${ }^{\#} \mathrm{P}<0.05$ vs GDM

GDM, which represents a particular group of hyperglycemic women. Our study shows that GDM has the same positively correlated as diabetes on the demand for opioid analgesics despite the brief period of elevated blood glucose.

Kim et al. showed that preoperative HbA1c is positively correlated with the postoperative opioid needs of patients with diabetes [3]. In our study, the patients in the GDM group were subgrouped based on whether their $\mathrm{HbA} 1 \mathrm{c}$ was $>6 \%$, given that the normal upper limit of $\mathrm{HbA} 1 \mathrm{c}$ is $6 \%$. However, we found no significant between-group differences in postoperative analgesia consumption, which was not showed in the results, most likely because the sample size was small (HbA1c was > $6 \%$ in only six GDM patients). Additional studies are needed to validate this hypothesis.

Notably, this study showed a significant betweengroup difference in the frequency of PCA use, indicating that the GDM group required significantly more postoperative analgesia than the NGDM group. Moreover, sufentanil consumption was significantly higher in the GDM group than in the NGDM group $6 \mathrm{~h}$ after surgery, with no significant between-group difference in VAS scores. Although sufentanil consumption did not differ significantly between the two groups at $24 \mathrm{~h}$, the significantly higher VAS scores and greater PCA use, which reflect maternal needs, suggest an increased opioid requirement in the GDM group that was limited by the "lock-out time" setting of the analgesic pump.

This cohort study matched conditions between the two groups with regard to factors such as weight and height that affect the demand for analgesics. Furthermore, previous studies have shown that contraction pain is more severe after a repeat cesarean section than after a first cesarean section [19]; therefore, parity was also used as a matching condition. Maternal age and gestational age were not matched. On the one hand, advanced age is a high-risk factor for GDM, and the gestational termination times at which pregnant women with GDM undergo cesarean section are earlier. On the other hand, no studies have indicated that maternal age or gestational week influences the demand for analgesics. Our results confirmed these suggestions, and a covariance analysis of the two variables showed that maternal age and gestational age did not affect the results.

This study has some limitations. First, GDM is diagnosed when two or more test values in the oral glucose tolerance test reach or exceed the reference value. The diagnostic criteria were strictly followed, and the patients were instructed to fast for 8 to $12 \mathrm{~h}$ prior to the glucose tolerance test. However, some patients might not have followed this instruction, leading to false positive results. We did not ask the patients to undergo retesting, although the inclusion of these patients would only reduce between-group differences. Second, this study only addressed analgesia by intravenous sufentanil after cesarean section. However, many hospitals routinely use intrathecal morphine and intravenous NSAIDs for postoperative analgesia. Whether GDM affects the analgesic effect of the latter drugs remains to be further studied. In addition, because of the design of the cohort study, a double-blind condition was not possible; however, the researchers responsible for the follow-up assessment were unaware of the study groups to which individual patients belonged, and the primary outcome was the objective analgesic pump data; thus, the objectivity of the research results was not affected. Finally, we recorded patient analgesic consumption up to $24 \mathrm{~h}$ after

Table 3 Covariance analysis of sufentanil consumption $6 \mathrm{~h}$ after surgery between two groups

\begin{tabular}{llllll}
\hline Source & Sum of Squares (Class III) & Degrees of Freedom & Square of Average & F-value & $P$-value \\
\hline Modified model & 261.558 & 3 & 87.186 & 1.998 & 0.124 \\
Intercept & 31.749 & 1 & 31.749 & 0.728 & 0.397 \\
Age & 38.968 & 1 & 38.968 & 0.893 & 0.348 \\
Gestational week & 0.216 & 1 & 0.216 & 0.005 & 0.944 \\
Group & 222.408 & 1 & 222.408 & 5.097 \\
Error & 2618.176 & 60 & 43.636 & & 0.028 \\
\hline
\end{tabular}


Table 4 Comparison of adverse reactions and patient satisfaction between two groups

\begin{tabular}{lll}
\hline & GDM $(n=32)$ & NGDM $(n=32)$ \\
\hline Nausea & $5(16)$ & $4(13)$ \\
Vomiting & $1(3)$ & $0(0)$ \\
Pruritus & $3(9)$ & $1(3)$ \\
Satisfaction $(1 / 2 / 3 / 4 / 5)$ & $0 / 0 / 3 / 27 / 2$ & $0 / 1 / 1 / 29 / 1$ \\
\hline
\end{tabular}

Data are number (\%)

surgery; we did not investigate later time points because we usually prescribe 24-h administration of routine intravenous analgesia given that patients typically start eating after $24 \mathrm{~h}$ and can take oral analgesics as needed. Moreover, milk production is usually low (mean: $<10$ $\mathrm{mL} / \mathrm{d}$ ) during the first 2 days after surgery and then begins to increase. Thus, the short-term use of regular doses of intravenous opioids after delivery results in extremely low drug concentrations in colostrum, with negligible drug intake among infants [20]. Based on these findings, 24-h postoperative intravenous analgesia is safe for nursing infants and helps mothers resume activities as soon as possible after delivery. The results of this study are only applicable to the early postoperative period after cesarean section; whether differences in postoperative long-term analgesia use also occur should be explored in future studies.

\section{Conclusion}

Pregnant women with GDM require more analgesics and exhibit higher sufentanil consumption during the immediate postoperative period after cesarean section than NGDM patients. Clinicians should focus on postoperative analgesia management in GDM patients to improve the effectiveness of postoperative analgesia and increase patient satisfaction.

\section{Abbreviations}

ASA: American Society of Anesthesiologists; GDM: Gestational diabetes mellitus; HbA1c: Glycated hemoglobin; NGDM: Non-GDM; PCA: Patientcontrolled analgesia; PCIA: Patient-controlled intravenous analgesia; SPSS: Statistical package for social science; VAS: Visual analog scale

\section{Acknowledgements}

We are extremely grateful to Jiangnan Wu MD of the Department of Clinical Epidemiology, Obstetrics and Gynecology Hospital of Fudan University for help with the statistical analysis of our results.

\section{Authors' contributions}

YC: study design, data collection, data analyses, and article drafting. GWL: interpretation of the results and data analyses. $\mathrm{HJ}$ : data collection. HS: study design, interpretation of the results, and article drafting. All authors have read, critically revised, and accepted the final manuscript. All authors read and approved the final manuscript.

\section{Availability of data and materials}

The datasets used and/or analyzed during the current study are available from the corresponding author on reasonable request.

\section{Ethics approval and consent to participate}

The Ethics Committee of the Obstetrics and Gynecology Hospital, Fudan University approved this study on June 2018, under the registration number: 218-47. All patients signed an informed consent document before the study.

\section{Consent for publication}

Not applicable.

\section{Competing interests}

The authors declare that they have no competing interests.

Received: 3 April 2019 Accepted: 30 December 2019

Published online: 09 January 2020

\section{References}

1. Karci A, Tasdogen A, Erkin Y, Aktaş G, Elar Z. The analgesic effect of morphine on postoperative pain in diabetic patients. Acta Anaesthesiol Scand. 2004;48:619-24

2. Koenig RJ, Peterson CM, Jones RL, Saudek C, Lehrman M, Cerami A Correlation of glucose regulation and hemoglobin Alc in diabetes mellitus. N Engl J Med. 1976:295:417-20.

3. Kim SH, Hwang JH. Preoperative glycosylated haemoglobin as a predictor of postoperative analgesic requirements in diabetic patients: a prospective observational study. Eur J Anaesthesiol. 2015;32:705-11.

4. Weiner DA, Murphy JP, Gwam C, Loughran GJ, Vulpis C, et al. Increased opioid consumption in diabetics with operative ankle fractures: a retrospective case-control study. Eur J Orthop Surg Traumatol. 2019 Aug; 29(6):1319-23.

5. Courteix C, Bourget P, Caussade F, Bardin M, Coudore F, Fialip J, Eschalier A. Is the reduced efficacy of morphine in diabetic rats caused by alterations of opiate receptors or of morphine pharmacokinetics? J Pharmacol Exp Ther. 1998:285:63-70.

6. Yeung RO, Savu A, Kinniburgh B, Lee L, Dzakpasu S, Nelson C, Johnson JA, Donovan LE, Ryan EA, Kaul P. Prevalence of gestational diabetes among Chinese and south Asians: a Canadian population-based analysis. J Diabetes Complicat. 2017;3:529-36.

7. Getahun D, Nath C, Ananth CV, Chavez MR, Smulian JC. Gestational diabetes in the United States: temporal trends 1989 through 2004. Am J Obstet Gynecol. 2008;198:525.

8. Ferrara A. Increasing prevalence of gestational diabetes mellitus: a public health perspective. Diabetes Care. 2007:30:S141-6.

9. Mackenzie RG, Trulson ME. Effects of insulin and streptozotocin-induced diabetes on brain tryptophan and serotonin metabolism in rats. J Neurochem. 1978;30:205-11.

10. Todorovic SM. Is Diabetic Nerve Pain Caused by Dysregulated Ion Channels in Sensory Neurons? Diabetes. 2015;64(12):3987-9.

11. Zhang HH, Hu J, Zhou YL, Qin X, Song ZY, Yang PP, Hu S, Jiang X, Xu GY. Promoted interaction of nuclear factor- $\mathrm{KB}$ with Demethylated Purinergic $\mathrm{P} 2 \mathrm{X} 3$ receptor gene contributes to neuropathic pain in rats with diabetes. Diabetes. 2015 Dec:64(12):4272-84.

12. Park SH, Bahk JH, Oh AY, Gil NS, Huh J, Lee JH. Gender difference and change of alpha(1)- adrenoceptors in the distal mesenteric arteries of streptozotocin-induced diabetic rats. Korean J Anesthesiol. 2011;61:419-27.

13. Nie JJ, Sun S, Huang SQ. Effect of oxycodone patient-controlled intravenous analgesia after cesarean section: a randomized controlled study. J Pain Res. 2017;10:2649-55.

14. Gul H, Yildiz O, Dogrul A, Yesilyurt O, Isimer A. The interaction between IL1 beta and morphine: possible mechanism of the deficiency of morphineinduced analgesia in diabetic mice. Pain. 2000:89:39-45.

15. Gullapalli S, Gurumoorthy K, Kaul CL, Ramarao P. Role of L-type Ca2t channels in attenuated morphine antinociception in streptozotocin-diabetic rats. Eur J Pharmacol. 2002;435:187-94.

16. Ibironke GF, Saba OJ. Effect of hyperglycemia on the efficacy of morphine analgesia in rats. Afr J Med Sci. 2006;35:443-5.

17. Hajializadeh Z, Esmaeili-Mahani S, Sheibani V, Kaeidi A, Atapour M, Abbasnejad M. Changes in the gene expression of specific G-protein 
subunits correlate with morphine insensitivity in streptozotocin-induced diabetic rats. Neuropeptides. 2010;44:299-304.

18. Kiguchi N, Ding H, Peters CM, Kock ND, Kishioka S, Cline JM, Wagner JD, Ko MC. Altered expression of glial markers, chemokines, and opioid receptors in the spinal cord of type 2 diabetic monkeys. Biochim Biophys Acta. 1863; 2017:274-83.

19. Ortner CM, Granot M, RichebelP CM, Bollag L, Landau R. Preoperative scar hyperalgesia is associated with post-operative pain in women undergoing a repeat Caesarean delivery. Eur J Pain. 2013;17:111-23.

20. Baka NE, Bayoumeu F, Boutroy MJ, Laxenaire MC. Colostrum morphine concentrations during postcesarean intravenous patient-controlled analgesia. Anesth Analg. 2002;94:184-7.

\section{Publisher's Note}

Springer Nature remains neutral with regard to jurisdictional claims in published maps and institutional affiliations.

Ready to submit your research? Choose BMC and benefit from:

- fast, convenient online submission

- thorough peer review by experienced researchers in your field

- rapid publication on acceptance

- support for research data, including large and complex data types

- gold Open Access which fosters wider collaboration and increased citations

- maximum visibility for your research: over $100 \mathrm{M}$ website views per year

At BMC, research is always in progress.

Learn more biomedcentral.com/submissions 